\title{
Comment on: the tumour microenvironment shapes innate lymphoid cells in patients with hepatocellular carcinoma
}

\author{
Lixia Xu ${ }^{1,2}$, Qingxia Yang ${ }^{1,2}$, Ce Tang ${ }^{2}$, Ming Kuang ${ }^{1,2,3}$ \\ ${ }^{1}$ Cancer Center, the First Affiliated Hospital, Sun Yat-sen University, Guangzhou, China; ${ }^{2}$ Institute of Precision Medicine, the First Affiliated \\ Hospital, Sun Yat-sen University, Guangzhou, China; ${ }^{3}$ Department of Hepatopancreatobiliary Surgery Center, The First Affiliated Hospital, Sun \\ Yat-sen University, Guangzhou, China \\ Correspondence to: Ming Kuang. Department of Hepatopancreatobiliary Surgery Center, the First Affiliated Hospital, Sun Yat-sen University, \\ Guangzhou 510080, China. Email: kuangm@mail.sysu.edu.cn. \\ Comment on: Heinrich B, Gertz EM, Schäffer AA, et al. The tumour microenvironment shapes innate lymphoid cells in patients with hepatocellular \\ carcinoma. Gut 2021. [Epub ahead of print]. doi: 10.1136/gutjnl-2021-325288.
}

Submitted Dec 08, 2021. Accepted for publication Dec 28, 2021.

doi: 10.21037/hbsn-21-519

View this article at: https://dx.doi.org/10.21037/hbsn-21-519

Hepatocellular carcinoma (HCC) is the fourth leading cause of cancer-related deaths worldwide (1). Resident immune cell populations within the liver, which is considered the largest immune organ, increase the pathological complexity of HCC (2). Innate lymphoid cells (ILCs), recently identified leukocytes, are a family of innate immune cells with similar characters to T lymphocytes. ILCs largely exist in intestinal mucosal tissue and regulate intestinal inflammation, mucosal defense, and tissue repair in gut disease processes (3). Unlike T cells, ILCs lack antigenspecific receptors; therefore, they cannot directly mediate antigen-specific responses (4). However, they can be activated by cytokines and/or natural cytotoxic receptors (such as NKp44) (5). ILCs are a highly heterogeneous cell population with high plasticity. Based on the expression of transcription factors and the production of different cytokines, ILCs can be divided into ILC1, ILC2, and ILC3, which are counterparts of the three main helper $\mathrm{T}$ cell subsets (Th1, Th2, and Th17) (6). Although ILCs can be detected in various tumor types, their specific contribution to tumor immunity is yet to be thoroughly elucidated.

In a recently published article in Gut, Heinrich et al. (7) discovered that ILC-controlling cytokines were differentially expressed in non-tumor and tumor tissues of patients with HCC. Among the differentially expressed cytokine genes, 3 cytokines (IL-1 $\beta$, IL-33, and TGF- $\beta$ ) were shown to affect the plasticity and function of ILCs. Specifically, it was shown that TGF- $\beta$ can affect the plasticity from NK cells to ILC1s, that IL-33 triggers an ILC2 response, and that IL-1 $\beta$ participates in the plasticity of ILC3s to ILC1s. IL-33 was found to be significantly associated with better overall survival in an HCC cohort from The Cancer Genome Atlas (TCGA) database. On the other hand, one previous study showed that IL-1 $\beta$ and IL23 could promote the differentiation from ILC1s to ILC 3s, while ILC3s could differentiate to ILC1s in the presence of IL-12 and IL-18 (8). These studies indicate that specific cytokines are crucial in regulating the plasticity and immune functions of ILCs.

The distribution of ILC subsets is not balanced in human tissues, including tumors, and their frequencies can differ significantly among individuals. Heinrich et al. used the simultaneous measurement of protein and RNA expression at the single-cell level to accurately identify the precise signatures of ILC subpopulations (7). They revealed a significant increase in ILC2s from non-tumor to tumor tissues in most of the patients with HCC analyzed in their study, while ILC3 exhibited an opposite trend (7). Furthermore, the ratio of ILC2 to ILC1 increased from non-tumor to tumor tissues in most patients (7). ILC2 can participate in the antitumor immune response by producing IL-5 and IL-13 to recruit eosinophils to the tumor site and restrict tumor growth (9). However, ILC2s have also been reported as a tumor accomplice in a variety of tumor environments, including breast cancer, gastric cancer, and prostate cancer, with their tumor-promoting functions 
attributed to their ability to produce IL-4 and IL-13 (10). ILC1s and conventional NK cells have also been shown to have a direct antitumor function, while both tumorsuppressing and tumor-promoting functions have been reported for ILC3s (11). In addition, it has been reported that IL-17 and IL-22 secreted by ILC3s contribute to the development of colorectal cancer (CRC) (12).

In contrast, one recent study reported on the protective role of ILC3 cells in CRC. The dysregulation of ILC3s can affect the adaptive immune function and promote tumor progression and resistance to immunotherapy in CRC (13). In short, ILCs are found in many cancer types, but there have been conflicting findings regarding their roles in different tumors.

In Heinrich et al., tumor-infiltrating ILC2s were found to accumulate in human HCC tissues, and higher infiltrations of ILC2s in HCC were correlated to better patient survival rates (7). In addition, patients with high levels of IL-33 expression in tumors exhibited longer survival and a higher ILC2/ILC1 ratio, potentially correlated with higher CD8 ${ }^{+}$ T cell killing activity (7). Similarly, in another study, ILC2 infiltration was associated with better outcomes in patients with pancreatic ductal adenocarcinoma, and the antitumor function of ILC2s was found to be dependent on ILC2activating IL-33 (14). Conversely, another study showed that a higher infiltration of less-mature KLRG1- ILC2 in HCC promoted tumor development, attributed to the possibility that ILC2s mediate immunosuppression in the tumor microenvironment through the KLRG1- ILC2/ CXCL2/neutrophil pathway (15). The diverse functions of ILC2s on tumor regulation may be caused by multiple tumor types or by the influence of complicated cytokine networks. Therefore, it is necessary to clarify ILC2s more accurately and explore ILC2-controlling cytokines in more depth.

Based on the findings of Heinrich et al. (7), we have a deeper understanding of ILC classification, plasticity, and biological function in HCC. The authors revealed that ILC2s, which are activated by IL-33, obtain antitumor properties in HCC, although the detailed mechanism was not clarified (7). Whether ILCs are present and functional in other tumors besides HCC is worth investigating. Many researchers have analyzed the regulatory effects of specific cytokines on ILCs, but whether ILCs interact with tumor microenvironment directly through expressing a range of surface molecules or are mediated by cytokines still needs further investigation. Future studies on ILCs and cancers should explore the specific biological functions and clinical significance of these heterogeneous plastic cells in the tumor microenvironment to contribute to the development of ILC-targeted strategies for immune intervention therapies.

\section{Acknowledgments}

Funding: None.

\section{Footnote}

Provenance and Peer Review: This article was commissioned by the editorial office, Hepatobiliary Surgery and Nutrition. The article did not undergo external peer review.

Conflicts of Interest: All authors have completed the ICMJE uniform disclosure form (available at https://hbsn. amegroups.com/article/view/10.21037/hbsn-21-519/coif). The authors have no conflicts of interest to declare.

Ethical Statement: The authors are accountable for all aspects of the work in ensuring that questions related to the accuracy or integrity of any part of the work are appropriately investigated and resolved.

Open Access Statement: This is an Open Access article distributed in accordance with the Creative Commons Attribution-NonCommercial-NoDerivs 4.0 International License (CC BY-NC-ND 4.0), which permits the noncommercial replication and distribution of the article with the strict proviso that no changes or edits are made and the original work is properly cited (including links to both the formal publication through the relevant DOI and the license). See: https://creativecommons.org/licenses/by-nc-nd/4.0/.

\section{References}

1. Villanueva A. Hepatocellular carcinoma. N Engl J Med 2019;380:1450-62.

2. Bray F, Ferlay J, Soerjomataram I, et al. Global cancer statistics 2018: GLOBOCAN estimates of incidence and mortality worldwide for 36 cancers in 185 countries. CA Cancer J Clin 2018;68:394-424.

3. Vivier E, Artis D, Colonna M, et al. Innate lymphoid cells: 10 years on. Cell 2018;174:1054-66.

4. Morita H, Moro K, Koyasu S. Innate lymphoid cells in allergic and nonallergic inflammation. J Allergy Clin Immunol 2016;138:1253-64.

5. Glatzer T, Killig M, Meisig J, et al. ROR $\gamma \mathrm{t}^{+}$innate 
lymphoid cells acquire a proinflammatory program upon engagement of the activating receptor NKp44. Immunity 2013;38:1223-35.

6. Spits H, Artis D, Colonna M, et al. Innate lymphoid cells-a proposal for uniform nomenclature. Nat Rev Immunol 2013;13:145-9.

7. Heinrich B, Gertz EM, Schäffer AA, et al. The tumour microenvironment shapes innate lymphoid cells in patients with hepatocellular carcinoma. Gut 2021. [Epub ahead of print]. doi: 10.1136/gutjnl-2021-325288.

8. Bernink JH, Krabbendam L, Germar K, et al. Interleukin-12 and -23 Control Plasticity of CD127(+) Group 1 and Group 3 Innate Lymphoid Cells in the Intestinal Lamina Propria. Immunity 2015;43:146-60.

9. Saranchova I, Han J, Zaman R, et al. Type 2 innate lymphocytes actuate immunity against tumours and limit cancer metastasis. Sci Rep 2018;8:2924.

10. Trabanelli S, Chevalier MF, Martinez-Usatorre A, et al. Tumour-derived PGD2 and NKp30-B7H6 engagement

Cite this article as: $\mathrm{Xu} \mathrm{L}$, Yang Q, Tang C, Kuang M. Comment on: the tumour microenvironment shapes innate lymphoid cells in patients with hepatocellular carcinoma. HepatoBiliary Surg Nutr 2022;11(2):280-282. doi: 10.21037/ hbsn-21-519 drives an immunosuppressive ILC2-MDSC axis. Nat Commun 2017;8:593.

11. Mattiola I, Diefenbach A. Innate lymphoid cells and cancer at border surfaces with the environment. Semin Immunol 2019;41:101278.

12. Kim J, Kim W, Moon UJ, et al. Intratumorally establishing type 2 innate lymphoid cells blocks tumor growth. J Immunol 2016;196:2410-23.

13. Goc J, Lv M, Bessman NJ, et al. Dysregulation of ILC3s unleashes progression and immunotherapy resistance in colon cancer. Cell 2021;184:5015-5030.e16.

14. Moral JA, Leung J, Rojas LA, et al. ILC2s amplify PD-1 blockade by activating tissue-specific cancer immunity. Nature 2020;579:130-5.

15. Xu X, Ye L, Zhang Q, et al. Group-2 innate lymphoid cells promote hcc progression through CXCL2neutrophil-induced immunosuppression. Hepatology 2021;74:2526-43. 